\title{
Heterocyclic synthesis with nitriles: Synthesis of pyridazine and pyridopyridazine derivatives.
}

\author{
Ana M. F. Oliveira-Campos*, Abdellatif M. Salaheldin, Lígia M. Rodrigues \\ Centro de Química, IBQF, Universidade do Minho, Campus de Gualtar, 4710-057 Braga, Portugal \\ amcampos@quimica.uminho.pt
}

Introduction

During the past few decades increasing interest in the synthesis and properties of pyridazines, pyridazinones, pyridopyridazines, and pyridopyridazinones has been observed.

Pyridopyridazines and pyridopyridazinones show wide spectrum of biological activities as described in the literature [1-4]. Both classes of substances are of interest as antiviral and cancer therapies, since their structures and properties resemble those of certain current pteridines and purines.

Recently pyridazinone nucleus has been extensively studied in the search for new and selective medicinal agents as drugs acting on the cardiovascular system [5,6]. It has been discovered that several aminopyridazinones showed remarkable biological activities; for example, nootropic (memory-improving) [7].

In contrast to the phthalazinones, their aza analogs, pyridopyridazinones, are as yet relatively little studied their synthesis, chemical properties and further applications. The primary reasons for this are the difficulties associated with the preparation of pyridine starting materials which can easily be transformed into pyridopyridazinones. The only general method for the synthesis of the system pyridopyridazinones involves the cyclization of $o$-acylpyridinecarboxylic acids [8] and their derivatives (ester [8] and disubstitute amides [9] or of methyl esters of o-cyanopyridinecarboxylic acids [10] with hydrazine and monosubstituted hydrazines).

Continuing our interest on the synthesis of pyridazinones [11,12] we present in this work their synthesis applying a method which was previously employed in only a few cases to the preparation of pyridazinones [13].

\section{Results and Discussion}

The literature showed that, preparation of compounds $\mathbf{1}$ and $\mathbf{2}$ could be obtained from cyclocondensation of arylhydrazonomalononitriles with malononitrile, 2-amino-1,1,3tricycanopropene, and 3-amino-2-cyanopent-2-ene dicarboxylate [14], [15] respectively (Scheme 1).
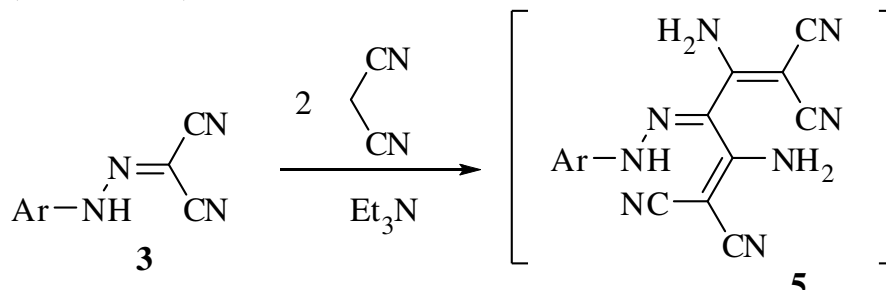<smiles>N#Cc1c(N)nc2c(C#N)c(=N)n([Al])nc2c1N</smiles><smiles>[Y][Y]([R14])([H])[N+](=O)OCC</smiles><smiles>[R]c1nc2c(c(N)c1C#N)C(=[R])N([Y19])N=C2C#N</smiles>

a, $\mathrm{R}_{1}=\mathrm{NH}, \mathrm{R}_{2} \mathrm{NH}_{2}$

2

b, $\mathrm{R}_{1}=\mathrm{O}, \mathrm{R}_{2}=\mathrm{OH}$

Scheme 1 
It was decided to build compounds of type 1 and 2 by both routes, from the reaction of 3-methoxyphenyl hydrazonomalononitrile with two equivalents of malononitrile (MN) and from coupling of arene diazonium chloride with 2-amino-1,1,3-tricycanopropene (MND), and 3-amino-2-cyanopent-2-ene dicarboxylate (ethyl or methyl cyano dimmer) to obtain pyridazine derivatives and further reaction of the latter with another mole of $\mathrm{MN}$ to obtain the desired products and confirm the way of cyclization of those compounds when reacted with $\mathrm{MN}$.

The reaction of compound 4a (MND), with aryldiazonium chlorides followed by cyclization in ethanolic triethylamine afforded the pyridazinimine derivatives 8a,b. Reaction of compounds 8a,b with another mole of malononitrile could takes place in two different pathways to afford pyrido[3,2-c]pyridazine or pyrido[2,3-d]pyridazine derivatives.

It was found that the reaction of pyridazine derivatives $\mathbf{8 a}, \mathbf{b}$ with malononitrile afforded $\mathbf{1 a}, \mathbf{b}$ in a good yield. Compound 1a,b could also be obtained from the reaction of $\mathbf{7 a , b}$ with malononitrile under refluxing in ethanolic triethylamine solution for $4 \mathrm{~h}$. The data in our hands, IR, mass, ${ }^{1} \mathrm{H}$ and ${ }^{13} \mathrm{C}$ NMR, are in accordance with the structure of pyrido[3,2-c] pyridazines 1 [15, cf. experimental part] (Scheme 2).

Moreover, the presence of an $\mathrm{NOE}$ enhancement between the $\mathrm{NH}$ group and $\mathrm{CH}$ aromatic protons in structure $\mathbf{1}$ and the absence of an NOE between $\mathrm{NH}$ and $\mathrm{NH}_{2}$ group for structure 2 allowed establishing the structure $\mathbf{1 .}$

The reaction of 2-(1-amino-2-cyanoethylidene)malonic acid diethyl $\mathbf{4 b}$ or dimethyl $\mathbf{4 c}$ ester and 4-chlorobenzene diazonium chloride to afford the hydrazo derivative 11a,b which could be cyclised into pyridazinone $\mathbf{1 2 a}, \mathbf{b}$. The reaction of compounds $\mathbf{1 2 a}, \mathbf{b}$ with malononitrile produce only pyridazine-3-carboxylic acid $\mathbf{1 3}$ which could also be obtained by boiling of compound $\mathbf{1 1}$ in ethanolic triethylamine solution for long time to furnish 13 (Scheme 2).

\section{Experimental}

All melting points were measured on a Gallenkamp apparatus and are uncorrected. IR spectra were determined on a Perkin Elmer FTIR-1600 using Nujol emulsions between $\mathrm{NaCl}$ plates. ${ }^{1} \mathrm{HNMR}$ spectra were recorded at $300 \mathrm{MHz}$ and $13 \mathrm{C}$ NMR spectra were determined at $75.4 \mathrm{MHz}$ both on Varian Unity plus spectrometer. In the NMR spectra was used TMS as internal reference. Low resolution EI mass spectra were determined on a Unicam GC-MS 120. FAB or high resolution mass spectra were obtained on a VG Ultima or AutoSpec E spectrometers. Elemental analysis data were obtained on a Leco CHNS-932.

\section{General procedure for the preparation of compounds 8a,b and 12a,b}

To a solution of each of $\mathbf{7 a}, \mathbf{b}$ and $\mathbf{1 1 a}, \mathbf{b}(10 \mathrm{mmol})$ in ethanol $(25 \mathrm{ml})$ was added triethylamine $(1 \mathrm{ml})$. The reaction mixture was refluxed for $2-6 \mathrm{~h}$, then allowed to cool, the precipitated solid products formed were collected by filtration, washed with cold water, and recrystalized from ethanol or ethanol/DMF (1:1) to afford 8a,b and 13a,b respectively. 

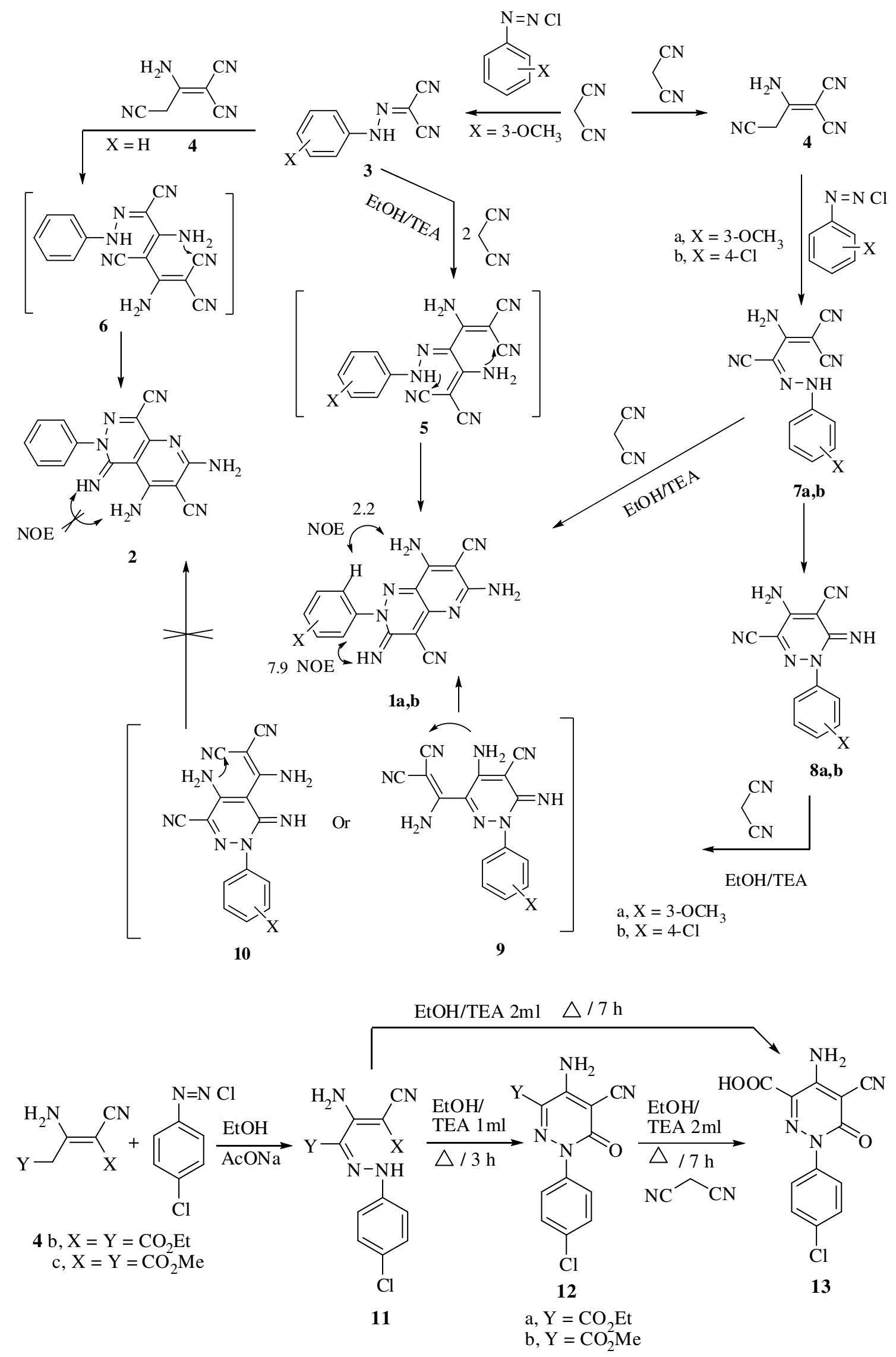

Scheme 2 
4-Amino-6-imino-1-(3-methoxyphenyl)-1,6-dihydro-pyridazine-3,5-dicarbonitrile (8a). Red powder, Yield $92 \%, \mathrm{mp}>300^{\circ} \mathrm{C}$ ethanol/DMF $(1: 1),{ }^{1} \mathrm{H}$ NMR $(300 \mathrm{MHz}$, DMSO-d $\left._{6}\right) \delta, 3.77\left(\mathrm{~s}, 3 \mathrm{H}, \mathrm{CH}_{3}\right), 7.04-7.08(\mathrm{~m}, 3 \mathrm{H}, \mathrm{Ar}-\mathrm{H}), 7.41-(\mathrm{s}, 1 \mathrm{H}, \mathrm{NH}), 7.43(\mathrm{~s}$, $1 \mathrm{H}, \mathrm{Ar}-\mathrm{H}), 7.48$ (br, $\left.2 \mathrm{H}, \mathrm{NH}_{2}\right)$. Anal. Calcd. for $\left(\mathrm{C}_{13} \mathrm{H}_{10} \mathrm{~N}_{6} \mathrm{O}\right): \mathrm{C}, 58.64 ; \mathrm{H}, 3.79 ; \mathrm{N}$, 31.56. Found C, 58.51, H, 4.01, N, 30.99.

4-Amino-1-(4-chlorophenyl)-6-imino-1,6-dihydro-pyridazine-3,5-dicarbonitrile

(8b). Red powder, Yield $90 \%, \mathrm{mp}>300^{\circ} \mathrm{C}$ ]Lit. $\mathrm{mp} \mathrm{mp}>300^{\circ} \mathrm{C} 16$ ], ethanol/DMF (1:1), ${ }^{1} \mathrm{H}$ NMR $\left(300 \mathrm{MHz}, \mathrm{DMSO}-\mathrm{d}_{6}\right) \delta, 6.82(\mathrm{~s}, 1 \mathrm{H}, \mathrm{NH}), 7.7(\mathrm{~d}, 2 \mathrm{H}, J=9 \mathrm{~Hz}, \mathrm{Ar}-\mathrm{H})$, 7.99 (d, 2H, $J=9 \mathrm{~Hz}, \mathrm{Ar}-\mathrm{H}), 8.68\left(\mathrm{~s}, 2 \mathrm{H}, \mathrm{NH}_{2}\right)$.

\section{4-Amino-1-(4-chloro-phenyl)-5-cyano-6-oxo-1,6-dihydro-pyridazine-3-carboxylic acid ethyl ester (12a)}

Yellow powder, Yield $92 \%, \mathrm{mp} 290-292^{\circ} \mathrm{C}$ ethanol/DMF (1:1), MS (EI), m/z = 318 $\left[\mathrm{M}^{+}, 24.8 \%\right], 320\left[(\mathrm{M}+2)^{+}, 5 \%\right]$

${ }^{1} \mathrm{H}$ NMR (300 MHz, DMSO-d $\left.{ }_{6}\right) \delta, 1.26\left(\mathrm{t}, 3 \mathrm{H}, J=7 \mathrm{~Hz}, \mathrm{CH}_{3}\right), 4.33$ (q, 2H, $J=7 \mathrm{~Hz}$, $\mathrm{CH}_{2}$ ), 7.51-7.60 (m, 4H, Ar-H), 8.20 (brs, $2 \mathrm{H}, \mathrm{NH}_{2}$ ).

\section{4-Amino-1-(4-chloro-phenyl)-5-cyano-6-oxo-1,6-dihydro-pyridazine-3-carboxylic acid methyl ester (12b) \\ Yellow powder, Yield $80 \%, \mathrm{mp} 279-280^{\circ} \mathrm{C}$ ethanol/DMF $(1: 1)$, \\ ${ }^{1} \mathrm{H}$ NMR (300 MHz, DMSO-d ${ }_{6}$ ) $\delta, 3.83$ (s, 3H, CH 3 ), 7.51-7.60 (m, 4H, Ar-H), 8.10 (s, $1 \mathrm{H}, \mathrm{NH}), 8.42(\mathrm{~s}, 1 \mathrm{H}, \mathrm{NH})$. \\ Anal. Calcd. for $\left(\mathrm{C}_{13} \mathrm{H}_{9} \mathrm{ClN}_{4} \mathrm{O}_{3}\right)$ : C, 51,25; H, 2,98; N, 18,39. Found C, 51.24; H, 3.03; N, 18.39.}

General procedure for preparation of compounds 1a,b

To a solution of each of $\mathbf{7 a}, \mathbf{b}$ and $\mathbf{8 a}, \mathbf{b}(10 \mathrm{mmol})$, malononitrile $(10 \mathrm{mmol})$ in ethanol $(25 \mathrm{ml})$ was added triethylamine $(1 \mathrm{ml})$. The reaction mixture was refluxed for $3 \mathrm{~h}$, then allowed to cool, the precipitated solid products formed were collected by filtration, washed with ethanol, and recystalized from ethanol/DMF (1:2) to afford 1a,b

6,8-Diamino-3-imino-2-(3-methoxyphenyl)-2,3-dihydropyrido[3,2-c]pyridazine-4,7dicarbonitrile (1a)

Red powder, Yield $94 \%, \mathrm{mp}>300^{\circ} \mathrm{C}$ ethanol/DMF (1:1), (Lit. $\left.\mathrm{mp}>360^{\circ} \mathrm{C}, 15\right)$, IR, $2217(\mathrm{CN}), 3355,3457\left(\mathrm{NH}, \mathrm{NH}_{2}\right)$

${ }^{1} \mathrm{H}$ NMR (300 MHz, DMSO-d $\left.\mathrm{d}_{6}\right) \delta, 3.79$ (s, 3H, $\mathrm{CH}_{3}$ ), 6.29 (brs, 1H, NH, exchangeable $\mathrm{D}_{2} \mathrm{O}$ ), $7.03(\mathrm{dd}, 1 \mathrm{H}, J=9 \mathrm{~Hz}, 2.1 \mathrm{~Hz}, \mathrm{Ar}-\mathrm{H}), 7.15(\mathrm{~m}, 2 \mathrm{H}, \mathrm{Ar}-\mathrm{H}), 7.43$ (t, $1 \mathrm{H}, J=9 \mathrm{~Hz}$, Ar- $\mathrm{H}$ ), 7.63 (brs, $1 \mathrm{H}, \mathrm{NH}$, exchangeable $\left.\mathrm{D}_{2} \mathrm{O}\right), 8.08$ (s, $2 \mathrm{H}, \mathrm{NH}_{2}$, exchangeable $\mathrm{D}_{2} \mathrm{O}$ ), 8.26 (brs, $1 \mathrm{H}, \mathrm{NH}$, exchangeable $\mathrm{D}_{2} \mathrm{O}$ ).

${ }^{13} \mathrm{C}$ NMR (75.4 MHz, DMSO-d $\left.{ }_{6}\right) \delta 56.4\left(\mathrm{CH}_{3}\right), 70.9(\mathrm{C}-7), 89.8(\mathrm{C}-4), 112.4\left(\mathrm{C}-2^{\prime}\right)$, $114.7(\mathrm{CN}), 114.8$ (C-4'), $115.9(\mathrm{CN}), 118.5$ (C-6'), 126.2 (C-9), 129.8 (C-5'), 141.8 (C-1'), 148.9 (C-9a), 154.3 (C-3), 155.4 (C-8), 159.7 (C-3'), 161.4 (C-6).

MS (FAB) $m / z 333\left(\mathrm{M}^{+}+1,100 \%\right)$,

Anal. Calcd. for $\left(\mathrm{C}_{16} \mathrm{H}_{12} \mathrm{~N}_{8} \mathrm{O}\right)$ : C, 57.83; H, 3.64; N, 33.72. Found C, 57.49, H, 4.02, N, 33.95 .

6,8-Diamino-2-(4-chlorophenyl)-3-imino-2,3-dihydropyrido[3,2-c]pyridazine-4,7dicarbonitrile (1b)

Red powder, Yield $90 \%, \mathrm{mp}>300^{\circ} \mathrm{C}$ ethanol/DMF (1:1), ${ }^{1} \mathrm{H}$ NMR $(300 \mathrm{MHz}$, DMSO$\left.\mathrm{d}_{6}\right) \delta, 6.50(\mathrm{~s}, 1 \mathrm{H}, \mathrm{NH}), 7.57(\mathrm{~d}, 2 \mathrm{H}, J=9 \mathrm{~Hz}, \operatorname{Ar}-\mathrm{H}), 7.68(\mathrm{~d}, 2 \mathrm{H}, J=9 \mathrm{~Hz}, \mathrm{Ar}-\mathrm{H})$, $7.69(\mathrm{~s}, 1 \mathrm{H}, \mathrm{NH}), 8.10$ (s, 2H, $\left.\mathrm{NH}_{2}\right), 7.69,8.29$ (s, 1H, NH). 


\section{4-Amino-1-(4-chlorophenyl)-5-cyano-6-oxo-1,6-dihydro-pyridazine-3-carboxylic acid 13}

To a solution of each of 11a,b and 12a,b $(10 \mathrm{mmol})$ in ethanol $(25 \mathrm{ml})$ was added triethylamine $(1 \mathrm{ml})$. The reaction mixture was refluxed for $7 \mathrm{~h}$, then allowed to cool, the precipitated solid product formed was collected by filtration, washed with ethanol, and recystalized from ethanol/DMF $(1: 1)$ to afford $\mathbf{1 3}$.

Reddish brown powder, $\mathrm{mp} 244-246^{\circ} \mathrm{C}(\mathrm{EtOH})$, in $74 \%$ yield.

${ }^{1} \mathrm{H}$ NMR (300 MHz, DMSO-d $\left.{ }_{6}\right) \delta, 7.52(\mathrm{~m}, 5 \mathrm{H}, 4 \mathrm{Ar}-\mathrm{H}, 1 \mathrm{H}, \mathrm{NH}), 8.16(\mathrm{~s}, 1 \mathrm{H}, \mathrm{NH})$, $10.47(\mathrm{~s}, 1 \mathrm{H}, \mathrm{COOH})$.

\section{Acknowledgements}

We thank Fundação para a Ciência e Tecnologia and FEDER (POCTI-SFA-3-686) and post-Doctoral grant for A. Salaheldin (SFRH/BPD/31490/2006) and Miss Elisa Pinto for obtaining the NMR, and elemental analyses data.

\section{References}

1. Oka, Y.; Omura, K.; Miyake, A.; Itoh, K.; Tomimoto, M.; Tada, N.; Yurugi, S. Chem. Pharm. Bull. 1975, 23, 2239 and references within.

2. Matsuura, I.; Yoneda, F; Nitta, Y. Chem. Pharm. Bull. 1966, 14, 1010.

3. Tigler, M.; Stanovnik, B. Azolo- and Azinopyridazines and Some Oxa and Thia Analogs. In Condensed Pyridazines Including Cinnolines and Phthalazines; Castle, R. N. Ed.; John Wiley \& Sons, Inc.: New York, 1973; pp. 968-1012 and references within.

4. Kricka, L. Pure Appl. Chem., 1996,68 (I 0). 1825- 1830.

5. Frank, H.; Heinisch, G. Pharmacologically Active Pyridazines Part I. In Progress in Medicinal Chemistry, Ellis, G.P., West, G. B., Eds.; Elsevier: Amsterdam, 1990; Vol 27, 1.

6. Frank, H.; Heinisch, G. Pharmacologically Active Pyridazines Part II. In Progress in Medicinal Chemistry, Ellis, G.P., Luscombe, D.K., Eds.; Elsevier: Amsterdam, 1992; Vol 29, 141.

7. Matyus, P. 3(2H)-Pyridazinones: Some recent aspects of synthetic and medicinal chemistry. J. Heterocycl. Chem. 1998, 35, 1075-1089.

8. Tigler, M.; Stanovnik, B. Azolo- and Azinopyridazines and Some Oxa and Thia Analogs. In Condensed Pyridazines Including Cinnolines and Phthalazines; Castle, R. N. Ed.; John Wiley \& Sons, Inc.: New York, 1973; pp. 968-1012 and references within.

9. Epsztajn, J.; Brzezifiski, J. Z.; Czech, K Monatsh. Chem. 1993, 124, 549.

10. Kormendy, K.; Kovfics, T.; Szulfigyi, J.; Ruff, F.; K6vesdi, I. Acta Chim. Hung. 1981, 108, 167.

11. Abdellatif M. Salah El-Din, Heteroatom chem. 2003, 14, 612.

12. Fathy M. Abdelrazek, Abdel-latif M. Salah El Din, and Ahmed E. Makky, Tetrahedron 2001, 57/9, 1813.

13. Fahmy, S. M.; Abded, N. M.; Mohareb, R. M.; Elnagdi, M. H. Synthesis, 1982, 490.

14. Kandeel, Z. E.; Abdelrazek, F. M.; Elnagdi, M. H.; Et-Torgoman, A. M. Heterocycles, 1982, 24, 2455.

15. Schäfer, H.; Gewald, K.; Gruner, M. J. Prakt. Chemie, 1989, 331, 878.

16. Hafez, E. A.; Khalifa, M. A.; Guda, S. K.; Elnagdi, M. H. Z. Naturforsch. 1981 $35 b, 485$. 